\title{
GLOBAL VARIANCE REDUCTION FOR MONTE CARLO \\ REACTOR PHYSICS CALCULATIONS
}

Qiong Zhang and Hany S. Abdel-Khalik

\begin{abstract}
The development of hybrid Monte-Carlo-Deterministic (MC-DT) approaches, taking place over the past few decades, have primarily focused on shielding and detection applications where the analysis requires a small number of responses, i.e. at the detector location(s). This work further develops a recently introduced global variance reduction approach, denoted by the SUBSPACE approach, and extends its application to reactor analysis problems, where responses are required everywhere in the phase space. In this proof-of-principle study, the Subspace approach is shown to reduce the excessively long execution time of Monte-Carlo reactor physics calculations for simplified reactor geometries significantly. By way of demonstration, the SUBSPACE approach is applied to assembly level calculations used to generate the few-group homogenized cross sections. These models are typically expensive and need to be executed in the order of $10^{3}-10^{5}$ times to properly characterize the fewgroup cross sections for downstream core-wide calculations. Applicability to keigenvalue core-wide models is also demonstrated in this work. Given the favorable results obtained in this work, we believe that the Subspace method significantly enhances the state of the art of Monte-Carlo reactor physics analysis with particular focus on reducing the necessary runtime for achieving accurate results.
\end{abstract}


Key Words: Hybrid Monte Carlo-Deterministic, Global Variance Reduction, FW-CADIS, SUBSPACE Approach 


\section{INTRODUCTION}

Monte Carlo (MC) methods have been applied in computing the fundamental mode of critical systems since the 1950s [1-4]. The use of Monte Carlo simulation in reactor design and analysis calculations has been promoted in recent years to preclude the need for the simplifying assumptions of deterministic methods [5]. However, among other obstacles (memory consumption, multi-physics feedback [6]), the excessively long runtime required for achieving accurate results and the slow convergence of the fission source preclude the application of the Monte-Carlo method for reactor analysis [7], [8]. For reducing the computational overhead of MC compared to deterministic models to manageable level, one must rely on global variance reduction (GVR) techniques to accelerate $\mathrm{MC}$ convergence. This follows as in the existing reactor physics methodology [8], the neutronic solver must be executed many times rendering $\mathrm{MC}$ simulations impractical for routine calculations. To demonstrate this situation, a short overview of the existing reactor physics methodology is given next. We reiterate that this paper addresses just one particular problem associated with the deployment of Monte-Carlo method for reactor physics analysis, but at least in the author's opinion it is the one of the more pressing issues. As pointed out in Refs. [6] and [9], the application of full-core Monte-Carlo reactor analysis on a regular basis is still not within reach of the reactor physics community and problems beyond acceleration have to be addressed before this final goal can be achieved. 
In current methodologies, the reactor core is divided up into smaller regions, often chosen to represent full or parts of a fuel assembly taken at different axial levels. Lattice physics calculations are used to analyze these regions in more detail, often done with many energy groups and fine spatial and angular mesh. The flux solution from lattice physics calculations is used to generate cross sections that are homogenized over coarser energy groups and spatially over each region in a manner that preserves reaction rates over the various regions. The few-group cross sections are then used in core-wide simulation where the geometrical, energy, and spatial details of the regions are now smeared which reduces the effective dimensionality of the core-wide problem. Given the reliance of the flux solution from lattice physics calculations on the isotopic concentration, the fuel temperature, the coolant temperature, voiding, and control rod insertion, etc., the few-group cross sections must be generated at a matrix of different conditions to enable one interpolate the correct value for core-wide simulation. This is a formidable task as for typical LWR models the number of these conditions are overwhelmingly large. Take for example a BWR model: one typically has of the order of 30 lattice designs, each depleted using lattice physics calculations to end of life with about 50 depletion steps. In addition, BWRs require subdividing the core in axial layers ( $\sim 10$ layers) and performing a distinct assembly calculation for each layer because of the change of the moderator density along the height of the reactor core. This set of calculations has to be repeated for various power, control rod insertion, Boron concentration, and temperature histories $\left(\mathrm{n}_{\mathrm{h}}>20\right)$. The total number of flux solutions for a typical BWR is $30 \mathrm{x} 50 \mathrm{x}$ 
$10 \mathrm{x} \mathrm{n}_{\mathrm{h}}=15,000 \mathrm{n}_{\mathrm{h}}$. If each flux solution takes in the order of few seconds, which is possible with highly customized commercial codes, these calculations can be completed over a short period of time. With MC models however, unless one has a reasonably fast convergence scheme, the use of $\mathrm{MC}$ would be infeasible for routine reactor physics calculations.

To address this challenge, variance reduction techniques have been developed to accelerate $\mathrm{MC}$ convergence. The idea is that if one has an approximate idea about the solution, one can use that knowledge to bias MC particles. For adjoint-based variance reduction techniques, which represent our current interest, a simplified deterministic model is used to calculate an adjoint flux for the response of interest, say a detector response placed somewhere in the reactor core. Given that the adjoint flux can be shown mathematically to describe the importance of particles at different points in the phase space, one can design weight window maps based on the adjoint information to bias MC particles. These weight windows are used for splitting particles that are important and playing Russian roulette with particles that are less important. The idea has been successfully demonstrated in the FW-CADIS methodology [7], which generalizes the idea of variance reduction to problems with global responses, i.e., that is when responses are desired everywhere in the phase space. This is done by employing an additional deterministic forward flux solution to assign more weight to regions with low flux and less weight to regions with high flux rendering a uniform variance reduction over all responses of interest. An assembly 
model represents such an example where the neutron flux is required everywhere in the assembly to properly homogenize the cross sections.

Over the past couple of years, a new approach, denoted by the SUBSPACE approach [8], was introduced to perform GVR with two primary advantages over existing FW-CADIS methodology. First, the forward flux solution is not required, which result in considerable time saving especially for eigenvalue problem with dominance ratio close to unity. Second, via the use of the so-called pseudo responses, representing random linear combinations of the original responses, the number of MC particles required to reach a given level of variance reduction is significantly reduced. Even though it is not a hallmark of the subspace method, we note that the creation of the importance maps can be performed in parallel and the number of Monte-Carlo particles can be split over multiple MC simulations thus increasing efficiency. This approach might be difficult with FWCADIS, where an expensive forward eigenvalue calculation must be performed. In order to achieve significant parallel speedup, the eigenvalue solver needs to be parallelized.

In previous work, the figure of merit (FOM) of the SUBSPACE approach was found to be in the range of 2-10 times faster than the FW-CADIS approach [8]. The lower range for the gain is for source-driven problems with small dimensionality in terms of the responses and the complexity of the geometry and energy details. The gain increases as the dimensionality of the model increases and reaches its maximum for eigenvalue core-wide problems. 
Throughout this work, the Subspace method's performance is compared to the FW-CADIS performance for a simplified full core reactor and assembly problem. It is noted, that apart from FW-CADIS and the SUBSPACE method other methods exist, that address the issue of slow MC convergence for reactor analysis. The Uniform Fission Site method (UFS) for example was shown to perform better than FW-CADIS for an NEA full core PWR MC benchmark [10]. However, the FW-CADIS method is chosen for comparison because its availability in the SCALE code package.

The purpose of this paper is to provide a proof-of-principle that the SUBSPACE method is a feasible approach to address the problems of slow convergence of Monte-Carlo reactor physics models.

\section{SUBSPACE APPROACH-BASED IMPLEMENTATION}

In its standard form, the k-eigenvalue transport equation is written as:

$$
\begin{array}{r}
{\left[\cdot \nabla+\sum_{T}(\vec{r}, E)\right](\vec{r}, E, \quad)=\iint\left(\vec{r}, E^{\prime}, \quad '\right) \sum_{s}\left(\vec{r}, E^{\prime} \rightarrow E, \quad \cdot \quad\right) d{ }^{\prime} d E^{\prime}} \\
+\frac{1}{k_{\text {eff }}} \frac{(E)}{4} \iint \sum_{F}\left(\vec{r}, E^{\prime}\right)\left(\vec{r}, E^{\prime},{ }^{\prime}\right) d \quad d E^{\prime}
\end{array}
$$

where the standard notations are used. The k-eigenvalue problem can conveniently be written in operator notation as:

$$
\mathrm{L}^{\rightarrow}=\frac{1}{k_{e f f}} F^{\rightarrow},
$$

The problem above is amenable to solution by Deterministic, analog MonteCarlo and hybrid Deterministic Monte-Carlo methods. Typically the user is interested 
in a set of responses given by the inner product of the forward flux solution ${ }^{-}$and a response function - of the form:

$$
u=\langle-,\rangle
$$

If only one response is sought, it is simple to tailor an adjoint function to this response. The adjoint function assigns importance values to different points in phase space based on their contribution to the response of interest:

$$
L_{i}^{*-*}=\frac{u_{i}}{-}={ }_{i}^{-},
$$

where $L^{*}$ is the adjoint transport operator and ${ }^{-*}$ is the importance map associated with this response. When applying the weight-window, a permissible range of weights can then be assigned to various regions in phase space based on this adjoint function: If the particle weight is below the specified range, it is Russian-rouletted and if it is above the range, it is split into multiple particles. In GVR problems, responses are desired everywhere, i.e.

$$
u_{i}=\left\langle-^{-},{ }_{i}\right\rangle, \text { and } i=1, \ldots, I
$$

where $I$ is the total number of responses, often representing the flux or reaction rates calculated at many regions in the phase space.

To ensure a uniform reduction of variances over the phase space, one needs to develop an adjoint function that helps bias MC particles towards all responses of interest [11]. In FW-CADIS, the adjoint source is formulated as a weighted sum of the individual responses' adjoint sources, weighted by the inverse of the forward flux [7], i.e. the following forward and adjoint problems are solved: 


$$
\begin{aligned}
& L^{-}=\frac{1}{k} F^{-} \quad u_{i}=\left\langle-{ }_{i}^{-}\right\rangle \\
& L^{*-*}={ }_{i=1}^{I} \frac{1}{u_{i}} \frac{u_{i}}{-}={ }_{i=1}^{I} \frac{-}{u_{i}},
\end{aligned}
$$

This approach is based on a sound engineering intuition in which more particles are sent to regions in the phase space where the flux is expected to be low and less particles to high flux regions. This helps render uniform variance reduction over all responses of interest.

In contrast, the SUBSPACE approach is based on a mathematical approach that takes advantage of the correlation between the various responses [8]. This helps reduce the effective number of responses for which weight windows are to be tailored. This is achieved by generating adjoint functions for the so-called pseudo-responses that are random linear combinations of the original responses:

$$
\tilde{u}_{j}={ }_{i=1}^{I}{ }_{i, j} u_{i}
$$

(where $\vec{\eta}_{j}$ is a randomly generated vector). Although, they lack physical meaning, these pseudo responses allow one to identify the minimum number of effective responses and their associated weight windows required to achieve the same level of variance reduction that would be achieved if all single-response weight window maps are employed to reduce variances for all responses one at a time (see Ref. [8] for details). The key reason for their success lies in the use of random numbers, which have been shown by nuclear researchers [8] and independently by mathematicians to 
help identify correlations/patterns in large data sets. Using the definition for $u_{i}$ from Eq. (4), one can write:

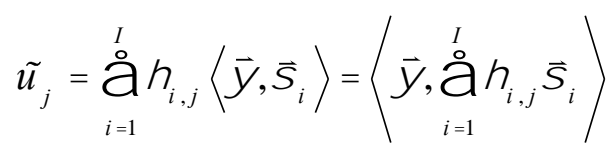

$$
\begin{aligned}
& L_{j}^{*} \sim_{j}^{*}=\frac{\tilde{u}_{j}}{-}={ }_{i=1}^{I}{ }_{i, j} \quad-
\end{aligned}
$$

In our context, the responses of interest are the few-group homogenized cross sections given by the general form:

$$
{ }_{x, g}=\frac{<{ }_{g}, x_{x, g}>}{g},
$$

where $x$ denotes the reaction type ( $x=$ fission, capture, scattering).

The pseudo response would correspondingly be described as:

$$
\tilde{u}_{x}=w_{x} w_{x, g}<{ }_{g}, x_{x, g}>
$$

where $x$ now stands for $x=$ fission, capture, scattering and flux and $w_{x, g}$ represents the weighting factor.

The adjoint source is constructed as:

$$
L_{x}^{*} \sim_{x}^{*}=\frac{\tilde{u_{x}}}{=}{ }_{g}{ }_{x} w_{x, g} \quad x, g
$$

Therefore, given the desired problem-specific cross section as the response of interest, the pseudo response is constructed as a linear combination of weighted original cross section responses from given libraries. For the SUBSPACE approach, 
the weighting factors are sampled from a statistical distribution and an algorithm is introduced to obtain the optimized number of correlations between given responses. For the FW-CADIS approach, the weighting factors are obtained from a forward calculation and calculated as the inverse of the response, in this case: the reaction rate. The algorithm to implement the SUBSPACE approach is described as follows:

\section{Requirements:}

- A general methodology that employs an importance map $\underset{i}{-*}$ to bias Monte Carlo particles towards a given response $u_{i}$.

- The capability to calculate an importance map ${ }_{j}^{\tilde{\sim}_{*}}$ for a pseudo response defined as a random linear combination of the original $I$ responses as defined in Eq. (11).

\section{Objective:}

- Identify, $r$ pseudo response, and employ them to reduce the variance for all $I$ responses.

\section{Algorithm:}

a) Estimate the rank $r$. If no prior knowledge about the rank is available, pick a small value, e.g. $5<r<20$, and execute step b. Calculate the Singular Value Decomposition (SVD) of the matrix containing the importance maps for the $r$

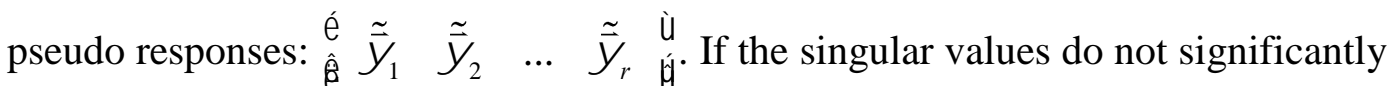
decline, increase the estimate for $r$.

b) PARALLEL DO $j=1, \ldots, r$

1. Generate a random vector 
2. Form a pseudo response $\tilde{u}_{j}={ }_{i=1}^{I}{ }_{i, j} u_{i}$

3. Calculate the importance map $\underset{i}{-*}$ associated with $\tilde{u}_{j}$

4. Bias Monte Carlo particles based on the $\begin{gathered}-* \\ i\end{gathered}$

5. Tally the original $I$ responses until the number of histories is exhausted

6. Record the responses $u_{i, j}^{\mu}$ and their standard deviations $u_{i, j}^{\sigma}$

\section{END DO}

c) COMBINE the responses and their standard deviations from the $r$ runs as follows:

$$
u_{i}^{\mu}=u_{i}^{\sigma} \sum_{j=1}^{r} \frac{u_{i, j}^{\mu}}{\left(u_{i, j}^{\sigma}\right)^{2}} \quad \text { and } \quad \frac{1}{u_{i}^{\sigma}}=\sum_{j=1}^{r} \frac{1}{\left(u_{i, j}^{\sigma}\right)^{2}}
$$

End Result:

- The $u_{i}^{\mu}$ and $u_{i}^{\sigma}$ are the mean and standard deviation for the $i^{\text {th }}$ response calculated by the SUBSPACE method.

This algorithm is composed of three steps. Step (a) requires an estimate of the rank $r$. Step (b) represents an execution of an existing variance reduction method with a special choice for the pseudo response. Since the importance function is often calculated using an adjoint model, this should be fairly easy to implement for most codes via simple manipulation of the right hand side of the adjoint equation. Next section provides more details on this step for incorporating the SUBSPACE method into the FW-CADIS framework. Step (c) combines the results from the $r$ executions, each with $N$ independent histories, under the assumption that they are statistically independent [8]. 
The adjoint flux map utilized for the SUBSPACE approach is generated using the three-dimensional discrete ordinates code DENOVO [11]. Similar to the FWCADIS approach, the optimization objective of the SUBSPACE approach is the calculation of the group-wise fluxes in fissionable regions [7]. In contrast to the FWCADIS eigenvalue implementation which requires the solution of a forward eigenvalue problem [12], the SUBSPACE approach solves a single adjoint fixed source problem with the adjoint source being determined as derivatives of the pseudo responses with respect to the adjoint function [8]. From the adjoint function the upper and lower weight window bounds are computed and subsequently the weight window map is written in a format suitable for MCNP [12]. The Monte-Carlo computations are all performed using MCNP.

If not stated otherwise, MCNP default settings are used. In particular for the weight window the following parameters are used:

1. Weight window ratio: $\mathrm{w}_{\mathrm{u}}=5$

2. Survival bias: $\mathrm{w}_{\mathrm{s}}=\left(\mathrm{w}_{\mathrm{u}} * 0.6\right) *($ Lower weight window bound $)$

3. Splitting parameter: $\mathrm{m}=50$ (Maximum particles after splitting).

For the weight cutoff, the default settings are used as well (one half of the minimum weight assigned to a source neutron). Finally, the maximum energy for physics cutoff is set to "very large" so that MCNP uses the cross sections' value at the maximum tabulated energy for particle energies that are above this energy. Further, neutrons are always treated by implicit capture. 
A simplified three-dimensional quarter core PWR model is employed to compare the performance of the FW-CADIS and the SUBSPACE approaches. The PWR quarter core model features a generic three-dimensional layout. The $x-y-z$ dimensions are $204.25 \times 204.25 \times 335.28 \mathrm{~cm}$. The model consists of $481 / 417 \times 17$ fuel assemblies (the $1 / 4$ assembly comes from dividing an odd number of assemblies along $\mathrm{x}$ and $\mathrm{y}$ by two when using symmetries to reduce the full core to a quarter core model), with 264 fuel rods per assembly each of which features a 3Wt\% U235/U uniform fuel enrichment. The geometry and spatial composition of this core are borrowed from Ref. [7].

The core geometry and composition is chosen for the purpose of providing a proof-of-principle that the SUBSPACE method is a feasible method for reducing the execution time of reactor physics Monte-Carlo calculations. The chosen geometry offers the advantage that is has already been used for testing FW-CADIS on a corewide eigenvalue calculation. It should be pointed out that results obtained for this benchmark might not translate exactly to other core geometries that are more heterogeneous. More realistic cores typically have a flatter power shape that might influence the performance of the SUBSPACE and FW-CADIS methods.

The adjoint fixed-source DENOVO calculations use an $S_{4}$ level symmetric quadrature and a $461 \times 461 \times 10$ spatial grid resolving the unit-cells. The 27 neutron and 19 photon energy group libraries included in the SCALE package are employed for generating the adjoint flux maps. The photon groups included in the SCALE 
library are excluded from the MCNP5 weight window input file and MCNP5 is used in "neutron" mode. For the flux and reaction rate responses, the first 14 neutron groups $(10.678 \mathrm{eV}<\mathrm{E}<20 \mathrm{MeV})$ define the fast group and the last 13 groups $(\mathrm{E}<3.059 \mathrm{eV})$ are thermal. A cross-sectional view of the model is presented in Fig. 1, where green represents moderator and reflector (water) and red the fuel pins.

Continuous-energy MCNP-5 simulations are conducted using 50,000 histories/cycle, 2500 active cycles with 500 inactive cycles starting from an initially uniform fission source. FW-CADIS and the SUBSPACE approaches are applied respectively and a thermal neutron flux energy bin from 0.15 to $0.275 \mathrm{eV}$ (to be consistent with the response selected by the FW-CADIS work, see Ref [7]) is selected to compare the performance of two approaches.

The distribution of relative uncertainties associated with the thermal neutron flux mesh tally (three-dimensional Cartesian array of tallies) in the given energy bin is plotted in Fig. 2 at the mid axial section of the core. Only the cells within the reactor core (i.e. excluding the reflector) are taken into account. The distribution of uncertainties obtained with the FW-CADIS and SUBSPACE approaches are similar demonstrating that for the same number of histories both methods obtain results featuring about the same level of confidence. The standard deviation and the mean value of the distribution of variances obtained with FW-CADIS and SUBSPACE approaches are presented in Table 1. For reference, the analog results are also provided. When applying the SUBSPACE approach, the obtained mean value of variance is 0.0181 ; while the mean value of variance obtained by performing FW- 
CADIS approach is 0.0346. Furthermore, the SUBSPACE approach generates a standard deviation of the variance distribution that is $33 \%$ lower compared to the same quantity generated by FW-CADIS implying a more uniform distribution of variances which is a good feature of variance reduction techniques.

The thermal neutron flux is chosen for comparison with the FW-CADIS method because it is a good indicator of accuracy of the fission and absorption rates. These reaction rates are dominated by large cross sections in the thermal range and hence the thermal neutron flux has a larger influence on their accuracy than the fast flux. Reaction rates are obtained by multiplying the computed flux with the known cross section value and summation over energy, and therefore the obtained flux from either method is the decisive factor in the methods' difference in performance. Therefore, for the presented comparison of FW-CADIS with the Subspace method, the thermal neutron flux is a good indicator of the difference of the methods' performance. The choice is also consistent with the FW-CADIS selection in Ref. [12]. In order to compare the efficiency, the global FOMs of the two approaches are calculated and presented in Table 2. It is important to note that the execution time of DENOVO when applying the SUBSPACE approach is $1 / 30$ compared to the execution time required by FW-CADIS. The primary reason for this is that the FWCADIS approach requires a forward eigenvalue flux solution [7] while the SUBSPACE approach only requires a source-driven solution. In most realistic corewide problems, the eigenvalue solution is very expensive because the dominance ratio is very close to 1.0 . 
The global FOM is calculated as the inverse product of the mean value of variance distribution and total execution time, which, in this case study, is the sum of the DENOVO execution time and the MCNP execution time. The global FOM is defined as:

$$
F O M=\frac{1}{\bar{v}\left(t_{D E N O V O}+{ }_{M C N P}\right)}
$$

For the analog MC simulation, the total time equals to the MCNP execution time. As shown in Table 2, the global FOM obtained from applying the SUBSPACE approach is 0.0649 while the global FOM of the FW-CADIS approach is 0.0071 . Meanwhile, the analog MC simulation has a global FOM value of 0.000698 . These results demonstrate that the SUBSPACE approach shows a nine-times speedup over FW-CADIS, which has been reported previously to show 6-10 speed up over analog MC. Finally, the SUBSPACE approach shows a speed-up of 93 over the analog.

\section{2 SENSITIVITY WITH RESPECT TO WEIGHT WINDOW PARAMETERS AND CHOICE OF DETERMINISTIC CROSS SECTION LIBRARY}

Within this section the sensitivity of the obtained statistical uncertainties within the mesh tally with respect to the three weight window parameters: weight window ratio, survival bias and splitting parameter are investigated. In addition, in order to determine the dependence of the results on the utilized cross section library in the deterministic calculation, the 44 group SCALE library is used instead of the 27 energy group libraries to compute the importance map. 
The results are presented relative to the "base case" $\left(\mathrm{w}_{\mathrm{u}}=5, \mathrm{w}_{\mathrm{s}}=0.6 * \mathrm{w}_{\mathrm{u}}\right.$, $\mathrm{m}=50$ ) using the following variations of parameters: $\mathrm{w}_{\mathrm{u}}=3,5,8 ; \mathrm{w}_{\mathrm{s}}=0.5,0.6,0.7 \mathrm{w}_{\mathrm{u}}$; $\mathrm{m}=5,50,500$. For each variation separately, the maximum relative difference $\varepsilon_{\max }$ and the RMS $\varepsilon_{\text {RMS }}$ of the uncertainties for the thermal neutron flux energy bin from 0.15 to $0.275 \mathrm{ev}$ are presented:

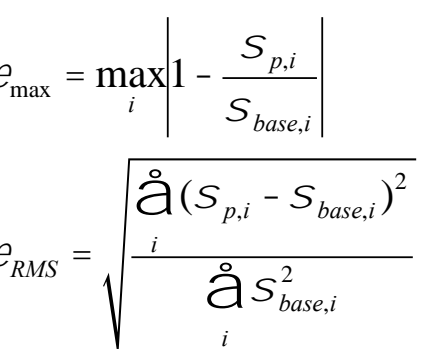

where $\sigma_{\mathrm{p}, \mathrm{i}}$ and $\sigma_{\mathrm{base}, \mathrm{i}}$ are the perturbed uncertainty for tally cell $\mathrm{i}$ and the base uncertainty of tally cell $i$, respectively.

The results are presented in Table 3 . The observed sensitivities with respect to $\mathrm{w}_{\mathrm{u}}, \mathrm{w}_{\mathrm{s}}, \mathrm{m}$, and the cross section library appear large when the difference of the results are measured in the maximum relative norm (>100\%), but are reasonably small when measured in the RMS norm $(\sim 5 \%)$. This indicates that the uncertainty of a single tally is sensitive to the choice of the weight window parameters, but in average the sensitivity of the overall performance averaged over the mesh is much smaller.

The potential reason is that depending on the course of the Monte-Carlo calculation, certain cells get more counts than other cells. Within a fission source generation, counts are accumulated and the fission sites are stored for starting particles in the next fission cycle. Fewer particles in one generation might lead to fewer particles throughout the whole Monte-Carlo simulation. When changing variance reduction parameters, regions with many fission sites might migrate in space 
leading to differences of the observed uncertainties. Another reason for the large maximum relative difference is that it might blows up even small absolute differences of two numbers if the base uncertainty is a small number itself.

Changing the cross section library for the deterministic calculation from 27 to 44 groups leads to comparable sensitivities as varying the weight window parameters. As increasing the number of groups increases the runtime of the deterministic calculation and the improvement in variance reduction is marginal, it seems not advisable to use the 44 group libraries instead of the 27 group libraries.

For the practitioner the variability is of little importance because the general characteristic of the uncertainty distribution does not change, just the position of cells with larger/smaller uncertainty.

\section{CROSS SECTION FUNCTIONALIZATION STUDY}

A peach-bottom seven-by-seven BWR assembly model is employed in this section [8]. The assembly model represents the southeast assembly of a typical $2 \times 2$ BWR control cell, which contains four assemblies and a cruciform control blade that is not modeled within the scope of this work. The specifications of the BWR assembly are listed in Table 4 . The assembly contains 49 fuel rods in a regular sevenby-seven fuel rod array. Each fuel pin is assigned with a unique fuel composition. The moderator around the fuel pin is separated into unit cells. The 49 unit cells are tallied and homogenized independently as shown in the Fig. 3 except when assembly homogenized cross sections are generated. 
The BWR model is implemented in the MCNP and NEWT computer codes. The keff computed by both codes differs by $360 \mathrm{pcm}$ and are therefore in good agreement. NEWT is a multi-group, discrete-ordinates radiation transport code that could be used to prepare collapsed cross sections and perform fixed-source and eigenvalue calculations [13]. NEWT also allows plenty of flexibility in defining boundary conditions [13]. The 44-group SCALE library is employed and collapsed into a thermal energy group and a fast energy group. The thermal group ranges from 0 through $0.625 \mathrm{eV}$ and the fast group comprises the energy range above $0.625 \mathrm{eV}$ [11]. Collapsed cross sections for each energy group are obtained from the 44-group library. An importance map is created based on a NEWT adjoint fixed-source calculation where the sources are constructed from the numerical values of the collapsed 44group cross section library. The importance map is consequently used in the MCNP calculation and for each material in the assembly to obtain the desired responses: functionalized cross sections are obtained.

The material representing unit cell one in the assembly serves as an example to compare the performance of different hybrid methods. The analog Monte Carlo simulation completes 2000 active cycles, 20000 histories per cycle in 351.02 minutes. When applying the SUBSPACE approach, the same number of histories is completed in 131.14 minutes and applying FW-CADIS it is completed in 99.72 minutes.

Three different cross section quantities are employed as examples to compare the performance: fission, capture and scattering cross sections. The relative uncertainties of the obtained numerical results are shown in Table 5 for thermal and 
fast energy groups. The SUBSPACE approach obtains an average uncertainty level that is $2 \sim 3$ times lower compared to the analog for the fast group and 4 5 times lower for the thermal group. Meanwhile, the SUBSPACE approach also shows a much better performance in reducing uncertainty compared to the FW-CADIS approach particularly for the thermal group, where a $50 \%$ decrease in term of relative uncertainty is obtained. This is due to the fact that the mean free path for the neutron in a thermal reactor is short which correlates each pin's responses to its nearest neighboring pins. Fast neutrons however have longer mean free path and they are able to reach the entire assembly from their birth to their death, hence the assembly features are more smeared for fast neutrons than they are for thermal neutrons. Since the thermal responses have more significant meaning compared to the fast flux in reactor physics, the SUBSPACE approach is particularly meaningful in realistic reactor analysis problems.

The results of the global FOM are shown in Table 6. The global FOM is calculated as:

$$
\begin{aligned}
& F O M_{G V R}=\frac{1}{\bar{v}\left(t_{N E W T}+t_{M C N P}\right)} \\
& F O M_{\text {ana } \log }=\frac{1}{\bar{v} t_{M C N P}}
\end{aligned}
$$

It is shown that for the thermal group, the SUBSPACE approach obtains a speedup between 32 38 over the analog; and for the fast group a speedup between 9 16 over the analog. On the other hand, the FW-CADIS approach obtains a speedup over the analog that is between 19 22 for the thermal group and a speed up between 6 11 for the fast group. Figures 4 and 5 plot the GVR methods' speedup for fast and 
thermal group for fission, capture and scattering independently. The blue bar and the red bar respectively identify the speedup over the analog of the SUBSPACE approach and the FW-CADIS approach. For all three reaction rates plotted in Figs. 4 and 5, the blue bar shows a more significant increase than the red bar, which demonstrates that the SUBSPACE approach is more efficient than the FW-CADIS approach in accelerating the analog MC procedure for both fast and thermal responses.

Typically, the reactor analyst is interested in assembly-homogenized cross sections. In order to demonstrate the efficiency of the SUBSPACE method for this type of application, assembly-homogenized fission, capture and scattering cross sections are obtained and compared among the three approaches: SUBSPACE, FWCADIS and analog Monte-Carlo.

The obtained speedups for fast and thermal groups are presented in Figs. 6 and 7 respectively. Even though the speedups follow the same trend as for the single fuel pin cross section study, the corresponding magnitude of the speedup is smaller. For the fast group a speedup of about 10 over analog Monte-Carlo is observed, while for the thermal group the speedup is about 20. Further, FWCADIS performs better in comparison with the SUBSPACE method than for the single fuel pin.

\section{DEPLETION STUDY}

The seven-by-seven BWR assembly model described in Section IV is employed in this section for demonstrating the feasibility of the SUBSPACE approach in the depletion calculation. The depletion calculation is conducted using the TRITON code sequence [11]. The 44 group energy library from SCALE, as 
introduced in Section IV, is employed and collapsed into thermal $0 \sim 0.625 \mathrm{eV}$ and fast $0.625 \mathrm{ev} \sim 20 \mathrm{MeV}$ groups. The 49 fuel pins are divided into eight groups based on the different composition for depletion purpose as shown in Fig. 8. The assembly model is simulated under hot condition with a pellet temperature of $900 \mathrm{~K}$ and a moderator temperature fixed at $600 \mathrm{~K}$. A constant power level of 45.220MW/MTU is maintained during the depletion that is split over five depletion cycles, each spanning a period of 100 days. Throughout the depletion cycles, the computed multiplication factor decreases from 1.08682 to 0.92166 . The Burnup level increases from $1.13 \mathrm{GWd} / \mathrm{MTU}$ to $19.2 \mathrm{GWd} / \mathrm{MTU}$.

The Monte Carlo calculations are performed using MCNP-5. The SUBSPACE approach is implemented for representing GVR methodology. Since the assembly level homogenized cross sections are chosen as the response of interest, the pseudo responses are constructed as a linear combination of weighted original cross section responses from the SCALE library. The analog Monte Carlo calculation is performed independently for comparison. For all depletion cycles, 20000 histories/cycle and 2000 active cycles are completed in the MCNP simulation. As demonstrated in [14], a single weight window in Monte Carlo simulation proves as accurate as multiple specified weight windows for all the depletion cycles. Therefore, to guarantee the maximum efficiency, in this work a single averaged weight window is constructed for all the depletion cycles employing the SUBSPACE approach. The number densities of nuclides are obtained for each depletion cycle from the TRITON calculation and different depletion scenarios are built. 
For each depletion scenario, an adjoint fixed-source problem, with the SUBSPACE pseudo response constructed by linearly combining original cross section data from the SCALE library, is solved and the corresponding importance map is obtained. Multiple importance maps (three to five per depletion cycle) are generated employing the SUBSPACE approach to represent the complete depletion process. All the importance maps are then linearly combined into one single importance map, based on which an "average" weight window is constructed for subsequent Monte Carlo simulations. To compare the performance of the SUBSPACE approach versus analog Monte Carlo, the figure of merits for group fluxes and reaction rates (fission, capture, scattering) at each depletion level are calculated as:

$$
\begin{aligned}
& F O M_{\text {SUBSPACE }}=\frac{1}{\bar{v}\left(t_{N E W T}+t_{M C N P}\right)} \\
& F O M_{\text {ana } \log }=\frac{1}{\bar{v} t_{M C N P}}
\end{aligned}
$$

For the SUBSPACE approach, the total time is computed as the sum of the deterministic calculation time from the adjoint fixed-source run by NEWT and the Monte Carlo calculation time from the MCNP simulation. For the analog, the total time is the Monte Carlo calculation time by MCNP. The final execution times of SUBSPACE and analog are listed in Table 7. The average time for all depletion runs is 451.84 minutes for the analog calculation and 149.14 minutes while applying the SUBSPACE approach.

In this study, flux and fission are employed as the two most important quantities to demonstrate the performance of the SUBSPACE approach. The corresponding FOM results are presented in Table 8 and Table 9. The speedups 
obtained by applying the SUBSPACE approach are plotted in Fig. 9 and Fig. 10 to show how the FOM speedups evolve throughout all the depletion cycles. For both flux and fission, the FOMs obtained by the SUBSPACE approach gain speedups that are between 40 50 for the thermal group and between 10 20 for the fast group when compared to the analog FOMs throughout all depletion cycles. Therefore, the SUBSPACE approach shows a more favorable performance regarding thermal responses as stated in Section 3 that the SUBSPACE approach is particularly efficient in accelerating thermal response calculations. In Fig. 9 and Fig. 10, it is shown that the speedups distribute evenly through the complete depletion process instead of showing an explicit increasing or decreasing trend. This well demonstrates the consistency of the performance of the single average weight window for all the depletion scenarios.

\section{CONCLUSION}

This work focuses on providing a proof-of-principle that the SUBSPACE method is a feasible method for addressing the slow convergence, and hence long execution times, of reactor physics Monte Carlo calculations. Two simplified test problems are utilized to show, by example, that the SUBSPACE method has great potential for future application in realistic reactor physics calculations. First, a full core eigenvalue problem was solved and comparison with the FW-CADIS method showed a speedup of nine. Second, a procedure was outlined conceptually that enables the use of Monte Carlo simulation to generate all few-group cross sections required for core-wide calculations. In particular, the generation of the few-group 
cross sections and the impact of depletion on the biasing procedure are investigated. Results indicate that the SUBSPACE approach results in 8-10 times speedup for fast group and 40-50 times speedup for thermal group over the analog, and the average weight window optimized to address all depletion cycles results in a consistent and effective speedup. In future work, it is proposed to extend this idea to investigate the impact of other core conditions on the weight windows, such as fuel temperature, coolant temperature, voiding, and control rod insertion. If all these changes result in insignificant loss of the speedup, the SUBSPACE approach will be extended to account for all core condition variations.

Furthermore, we will investigate the use of the GPT (Generalized Perturbation Theory)-free methodology to reduce the computational cost required to generate all the depletion and branch cases. Based on recent results of applying GPT-free to a realistic assembly models, we expect an additional significant speed up, since the GPT-free methodology allows one to directly calculate the change in the few-group cross sections due to changes in core parameters without having to re-execute the Monte Carlo model.

\section{ACKNOWLEDGEMENT}

This work has been supported by a Department of Energy Nuclear Energy University Program grant entitled 'Development of Subspace-Based Hybrid Monte Carlo-Deterministic Algorithms for Reactor Physics Calculations'. 


\section{REFERENCES}

1. E.L. KAPLAN, "Monte Carlo Methods for Equilibrium Solutions in Neutron Multiplication", UCRL-5275-T, Livermore National Laboratory (1958).

2. W. GOAD and R. JOHNSTON, "A Monte Carlo Method for Criticality Problems", Nucl. Sci. Eng.5, 371-375 (1959).

3. J. LIEBEROTH, "A Monte Carlo Technique to Solve the Static Eigenvalue Problem of the Boltzmann Transport Equation," Nukleonik 11,213 (1968).

4. M. R. MENDELSON, "Monte Carlo Criticality Calculations for Thermal Reactors," Nucl. Sci Eng. 32, 319-331 (1968).

5. K. SMITH, “Assembly Homogenization Techniques for Light Water Reactor Analysis," Progress in Nuclear Energy, 17, 3 (1986).

6. W.R. Martin, "Challenges and Prospects for Whole-Core Monte Carlo Analysis”, Nuclear Engineering and Technology, 44, 2, (2012).

7. J. C. WAGNER, S. W. MOSHER, "Forward Weighted CADIS Method for Variance Reduction of Monte Carlo Reactor Analysis,” Trans. Am. Nucl. Soc. 103. 342-344 (2010).

8. Z. WU, Q. ZHANG and H.S. ABDEL-KHALIK, "On Hybrid Monte CarloDeterministic Methods for Reactor Analysis", Nuclear Technology, In print, Dec. 2012.

9. K. Smith, "Challenges in the Development of High-Fidelity LRW Core Neutronics Tools”, M\&C 2013, Sun Valley, Idaho, May 5-9, (2013). 
10. D.J. Kelly, T.M. Sutton and S.C. Wilson, "MC21 Analysis of the Nuclear Energy Monte Carlo Performance Benchmark Problem”, Physor 2012: Advances in Reactor Physics - Linking Research, Industry and Education, (2012).

11. SCALE: A Modular Code System for Performing Standardized Computer Analyses for Licensing Evaluations, ORNL/TM-2005/39, Version 6, Vols. I-III, January 2009.

12. J.C. Wagner et al., "Hybrid and Parallel Domain-Decomposition Methods Development to Enable Monte-Carlo for Reactor Analyses", Progress in Nuclear Science and Technology, Vol. 2, pp. 815-820, (2011).

13. M.D. DEHART, "NEWT: A new transport algorithm for two-dimensional discrete ordinates analysis in non-orthogonal geometries", ORNL/TM-2005/39, UTBattelle, LLC, Oak Ridge National Laboratory, January (2009).

14. Q. Zhang and H. Abdel-Khalik, "Global Variance Reduction for Monte Carlo Reactor Physics Calculations”, ANS Winter Meeting, Nov. 11-15, (2012).

15. MCNP: A General Monte Carlo N-Particle Transport Code, Version 5, Volume 2: User's Guide.

16. F.B. BROWN, A Review of Monte Carlo Criticality CalculationsConvergence, Bias, Statistics, Monte Carlo Codes, X-3-MCC, LANL, M\&C, May 37, Saratoga (2009).

17. Q. ZHANG and H. S. ABDEL-KHALIK, "Adjoint-Based Global Variance Reduction Approach for Reactor Analysis Problems," Proc. Int. Conf. Math. Comp., Rio De Janeiro (2011). 
18. J. C. WAGNER, E. D. BLAKEMAN, and D. E. PEPLOW, "ForwardWeighted CADIS Method for Variance Reduction of Monte Carlo Calculations of Distributions and Multiple Localized Quantities," Proc. Int. Conf. Math. Comp. Saratoga Springs, NY (2009).

19. A. HAGHIGHAT, J. C. WAGNER, "Monte Carlo Variance Reduction with Deterministic Importance Functions," Progress in Nuclear Energy, Vol 42, No. 1 (2003).

20. J. C. WAGNER, E. D. BLAKEMAN, D.E. PEPLOW, "Forward-Weighted CADIS Method for Global Variance Reduction," ANS Annual Meeting, November 11-15, Washington, DC (2007).

21. J. C. WAGNER, D. E. PEPLOW, S. W. MOSHER, T. M. EVANS, "Review of Hybrid (Deterministic/Monte Carlo) Radiation Transport Methods, Codes, and Applications at Oak Ridge National Laboratory,” Joint International Conference on Supercomputing in Nuclear Applications and Monte Carlo 2010, Hitotsubashi Memorial Hall, Tokyo, Japan (2010).

22. Q. ZHANG and H. S. ABDEL-KHALIK, "Hybrid Monte Carlo-Deterministic Approach Global Variance Reduction," Transactions of the American Nuclear Society, Hollywood Florida (2011). 


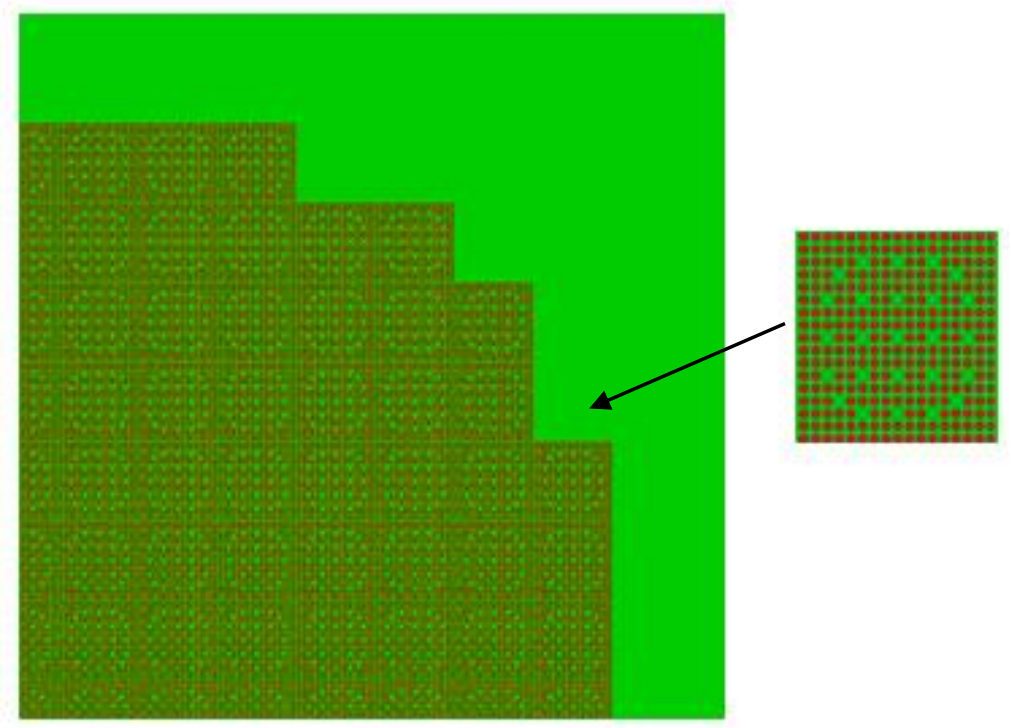

Fig.1. A Section View of 3-D PWR Quarter Core Model 

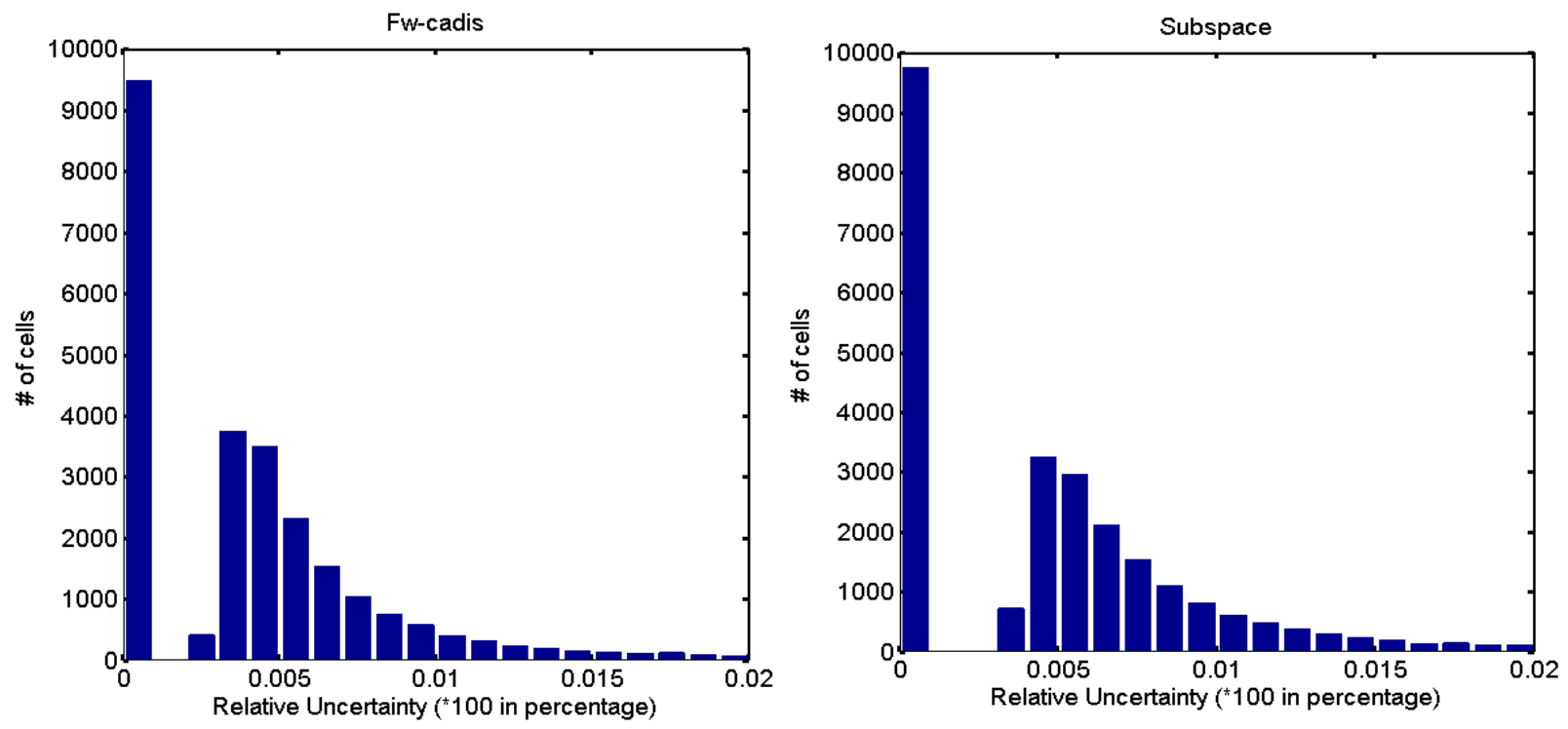

Fig. 2. Relative Uncertainty Distribution for Tallied Thermal Flux using FW-CADIS and SUBSPACE Approaches

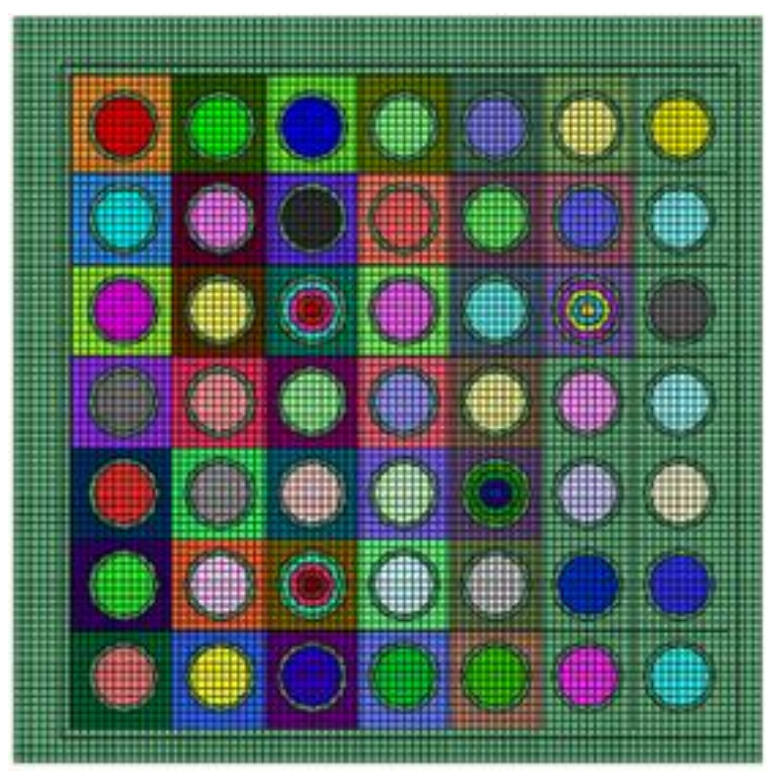

Fig.3. BWR Assembly Model 


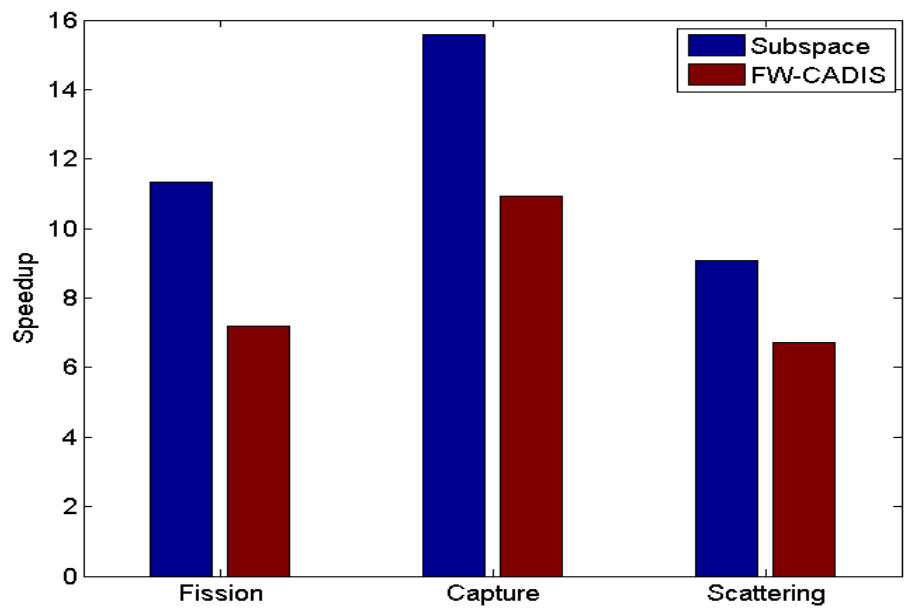

Fig.4. GVR Calculation Speedup for Fast Group

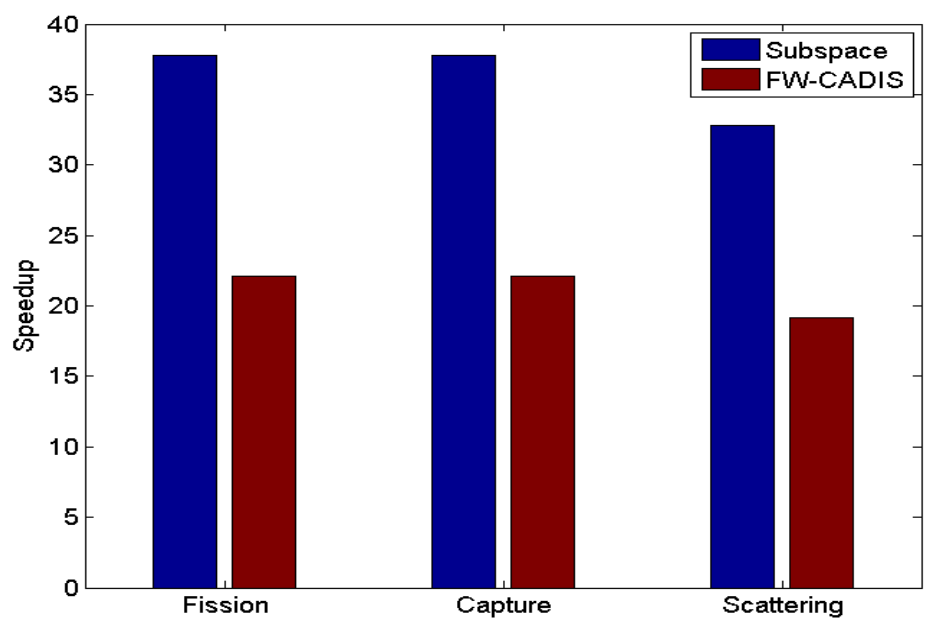

Fig.5. GVR Calculation Speedup for Thermal Group 


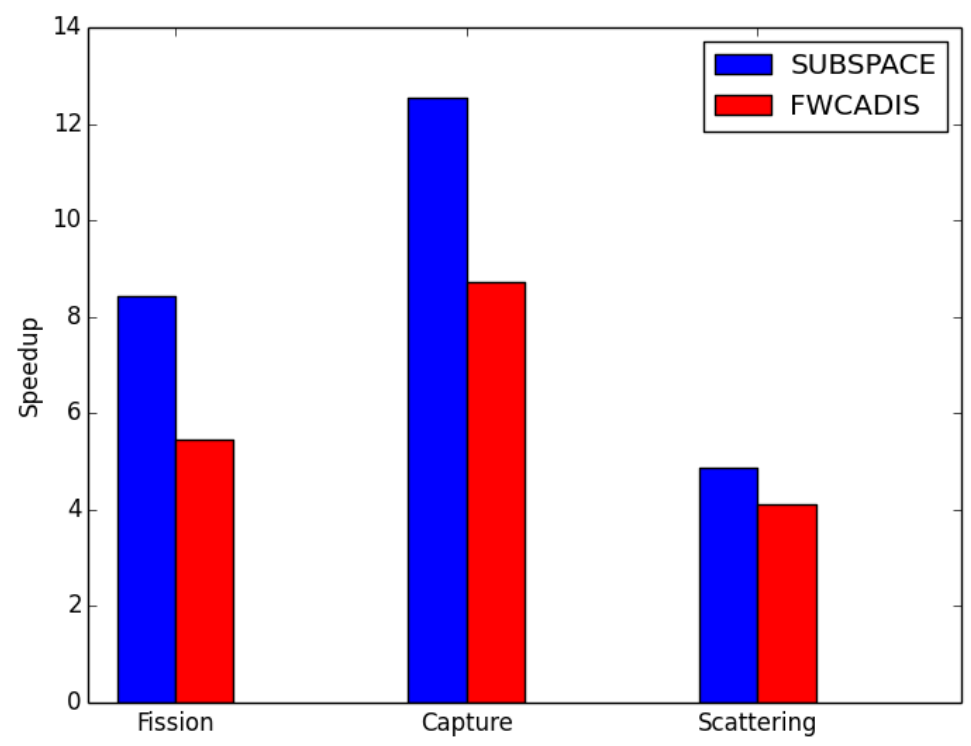

Fig.6: GVR full assembly-homogenized cross section calculation speedup for fast group.

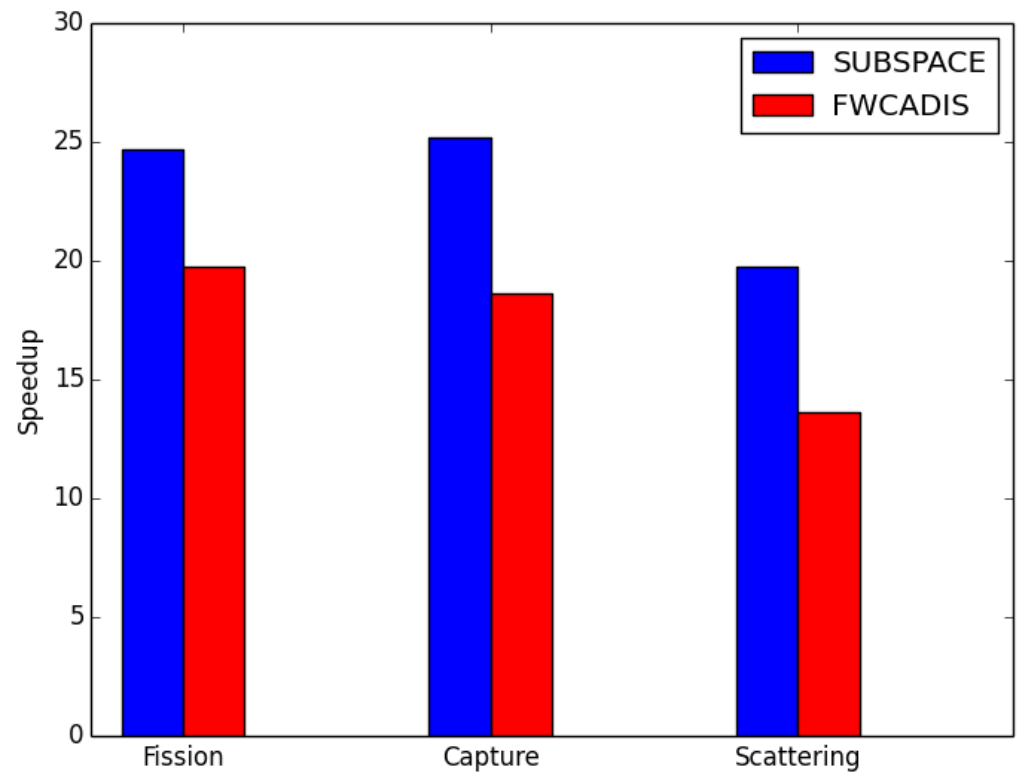

Fig.7: GVR full assembly-homogenized cross section calculation speedup for thermal group. 


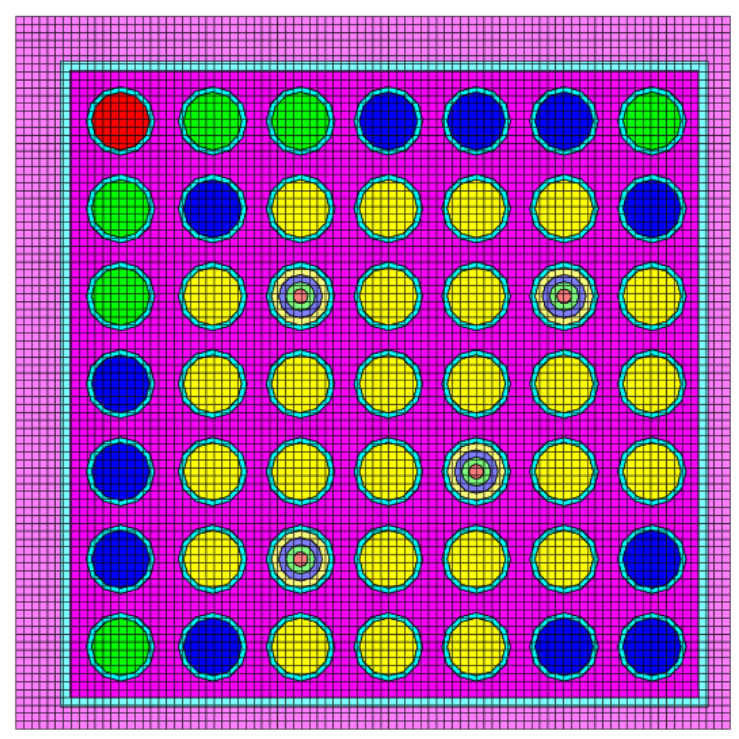

Fig.8: Pin Cells of BWR Assembly Model for Depletion

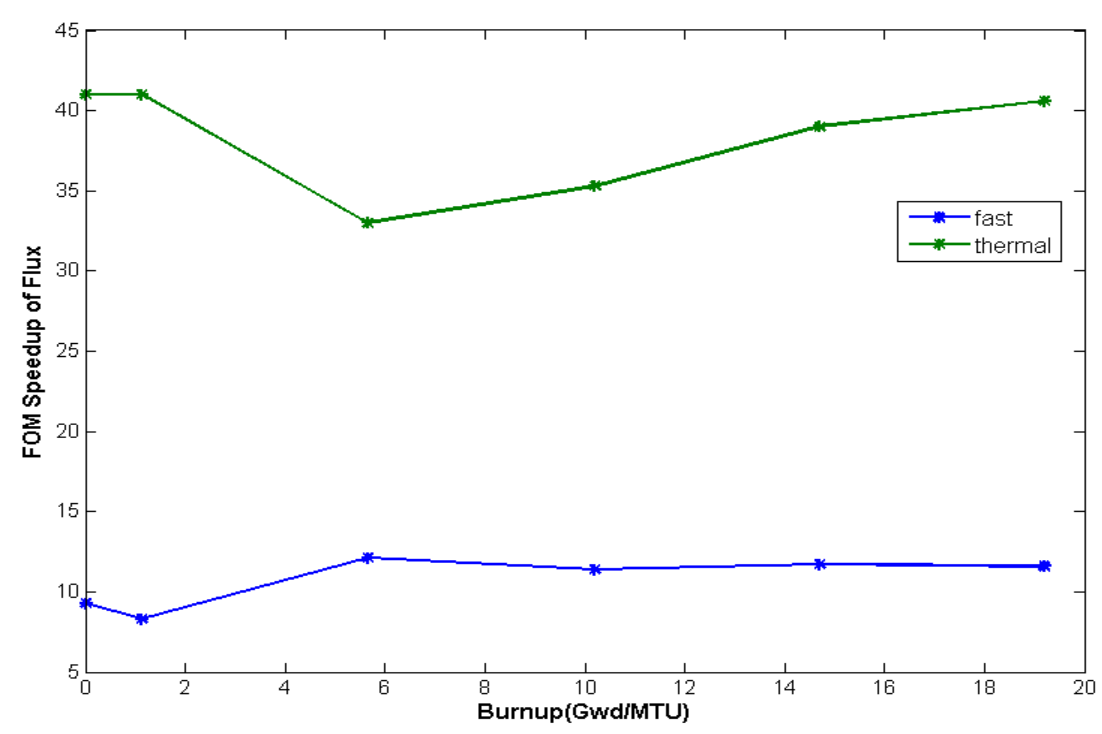

Fig.9: FOM Speedup for thermal and fast flux throughout Depletion Steps 


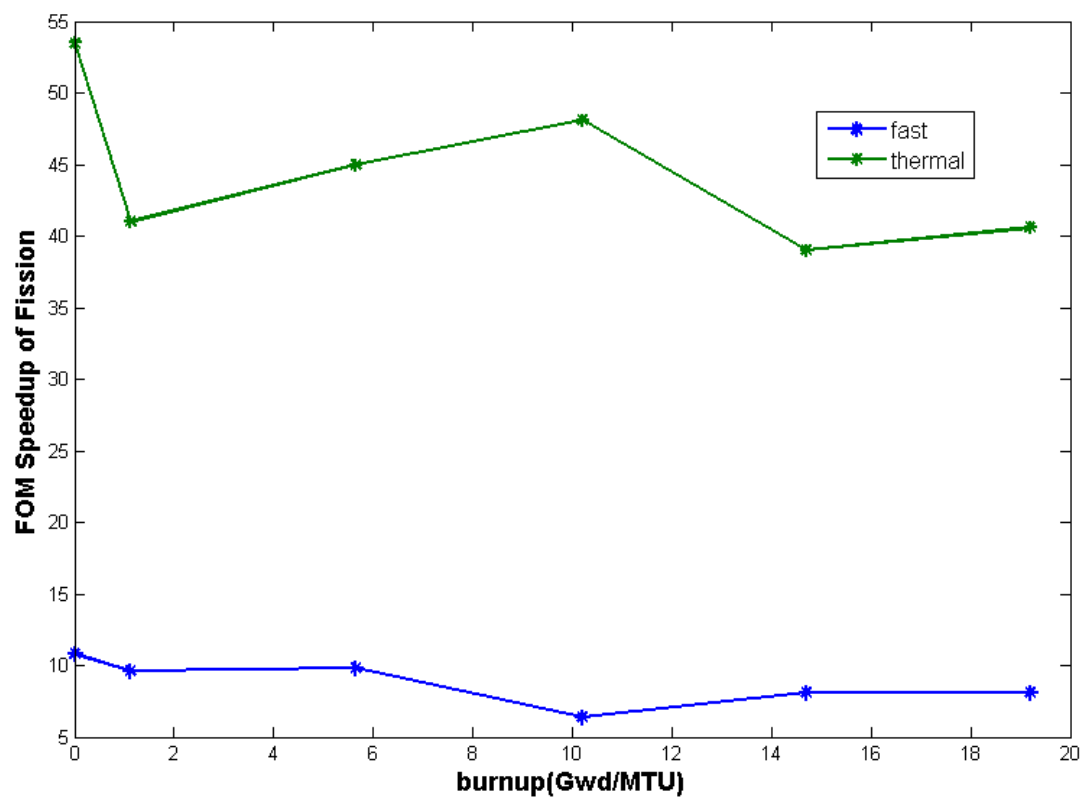

Fig.10: FOM Speedup of Fission throughout Depletion Steps. 
Table 1: Standard Deviation and Mean Value of the Variance Distribution

\begin{tabular}{|c|c|c|}
\hline & St dev of variance & Mean of variance \\
\hline FW-CADIS & 0.1503 & 0.0346 \\
\hline SUBSPACE & 0.1017 & 0.0181 \\
\hline Analog & 0.2145 & 0.0588 \\
\hline
\end{tabular}

Table 2: Execution Time and Global FOM

\begin{tabular}{|c|c|c|c|c|}
\hline \multicolumn{4}{|c|}{ Execution Time (mins) } & \multirow[t]{2}{*}{ FOM } \\
\hline & Denovo & MCNP & Total & \\
\hline SUBSPACE & 90.83 & 761.76 & 852.59 & 0.0649 \\
\hline FW-CADIS & 2883.05 & 1208.91 & 4091.96 & 0.0071 \\
\hline Analog & N/A & 24364.05 & 24364.05 & 0.000698 \\
\hline
\end{tabular}

Table 3: Sensitivities of the uncertainty distribution based on weight window parameters and utilized deterministic cross section library.

\begin{tabular}{|c|c|c|}
\hline \multicolumn{3}{|c|}{ Sensitivity with respect to wu } \\
\hline Parameter wu & $\varepsilon \mathrm{RMS}(\%)$ & $\operatorname{smax}(\%)$ \\
\hline 3 & 4.0 & 165.7 \\
\hline 8 & 5.1 & 73.4 \\
\hline \multicolumn{3}{|c|}{ Sensitivity with respect to ws } \\
\hline Parameter ws & $\varepsilon \mathrm{RMS}(\%)$ & $\operatorname{smax}(\%)$ \\
\hline $0.5 * \mathrm{wu}$ & 3.8 & 156.6 \\
\hline $0.7 * \mathrm{wu}$ & 2.0 & 168.1 \\
\hline \multicolumn{3}{|c|}{ Sensitivity with respect to $\mathrm{m}$} \\
\hline Parameter ws & $\varepsilon \mathrm{RMS}(\%)$ & $\operatorname{smax}(\%)$ \\
\hline 5 & 2.4 & 147.9 \\
\hline 500 & 2.41 & 165.5 \\
\hline \multicolumn{3}{|c|}{ Sensitivity with respect to cross section library } \\
\hline Number of groups & $\varepsilon \mathrm{RMS}(\%)$ & $\operatorname{cmax}(\%)$ \\
\hline 44 groups & 3.8 & 89.4 \\
\hline
\end{tabular}


Table 4: BWR Model Specification

\begin{tabular}{|c|c|}
\hline Assembly Pitch $(\mathrm{cm})$ & 15.24 \\
\hline Fuel Pitch $(\mathrm{cm})$ & 1.8745 \\
\hline Fuel Rod Diameter $(\mathrm{cm})$ & 1.2116 \\
\hline Cladding Thickness $(\mathrm{cm})$ & 0.1092 \\
\hline Canning Thickness $(\mathrm{cm})$ & 0.2032 \\
\hline Material Temperature (K) & 552.833 \\
\hline
\end{tabular}


Table 5: Relative Uncertainty of Homogenized Cross Sections

\begin{tabular}{|c|c|c|c|c|c|c|}
\hline & \multicolumn{3}{|c|}{ Fast Group (\%) } & \multicolumn{3}{c|}{ Thermal Group (\%) } \\
\hline & Analog & SUBSPACE & FW-CADIS & Analog & SUBSPACE & FW-CADIS \\
\hline Fission & 0.103 & 0.050 & 0.072 & 0.106 & 0.028 & 0.042 \\
\hline Capture & 0.206 & 0.085 & 0.117 & 0.106 & 0.028 & 0.042 \\
\hline Scattering & 0.078 & 0.042 & 0.057 & 0.099 & 0.028 & 0.042 \\
\hline
\end{tabular}

Table 6: Global FOM of Homogenized Cross Sections

\begin{tabular}{|c|c|c|c|c|c|c|}
\hline & \multicolumn{6}{|c|}{ Fast Group } \\
\hline & Analog & SUBSPACE & Speed-up & Analog & FW-CADIS & Speed-up \\
\hline Fission & $2.68 \mathrm{E}+03$ & $3.05 \mathrm{E}+04$ & 11.35 & $2.68 \mathrm{E}+03$ & $1.92 \mathrm{E}+04$ & 7.18 \\
\hline Capture & $6.70 \mathrm{E}+02$ & $1.04 \mathrm{E}+04$ & 15.58 & $6.70 \mathrm{E}+02$ & $7.31 \mathrm{E}+03$ & 10.92 \\
\hline \multirow[t]{3}{*}{ Scattering } & $4.67 \mathrm{E}+03$ & $4.23 \mathrm{E}+04$ & 9.07 & $4.67 \mathrm{E}+03$ & $3.13 \mathrm{E}+04$ & 6.71 \\
\hline & \multicolumn{6}{|c|}{ Thermal Group } \\
\hline & Analog & SUBSPACE & Speed-up & Analog & FW-CADIS & Speed-up \\
\hline Fission & $2.52 \mathrm{E}+03$ & $9.53 \mathrm{E}+04$ & 37.81 & $2.52 \mathrm{E}+03$ & $5.57 \mathrm{E}+04$ & 22.10 \\
\hline Capture & $2.52 \mathrm{E}+03$ & $9.53 \mathrm{E}+04$ & 37.81 & $2.52 \mathrm{E}+03$ & $5.57 \mathrm{E}+04$ & 22.10 \\
\hline Scattering & $2.90 \mathrm{E}+03$ & $9.53 \mathrm{E}+04$ & 32.79 & $2.90 \mathrm{E}+03$ & $5.57 \mathrm{E}+04$ & 19.16 \\
\hline
\end{tabular}


Table 7: Total Execution Time of Depletion Calculations

\begin{tabular}{|c|c|c|}
\hline \multirow{2}{*}{ Depletion Cycle } & \multicolumn{2}{|l|}{ Total Execution Time (mins) } \\
\cline { 2 - 3 } & Analog & SUBSPACE \\
\hline 1 & 402.6 & 120.3 \\
\hline 2 & 421.6 & 135.2 \\
\hline 3 & 441.8 & 146.1 \\
\hline 4 & 472.1 & 165.2 \\
\hline 5 & 521.1 & 178.9 \\
\hline
\end{tabular}


Table 8: The FOM Comparison of Flux

\begin{tabular}{|c|c|c|c|c|c|c|}
\hline \multirow{3}{*}{$\begin{array}{c}\text { Depletion } \\
\text { Cycle }\end{array}$} & \multicolumn{4}{|c|}{ FOM } & \multicolumn{2}{|c|}{ SUBSPACE Speedup } \\
\hline & \multicolumn{2}{|c|}{ Analog } & \multicolumn{2}{|c|}{ SUBSPACE } & \multirow[t]{2}{*}{ Fast } & \multirow[t]{2}{*}{ Thermal } \\
\hline & Fast & Thermal & Fast & Thermal & & \\
\hline No depletion & 9934.2 & 5068.5 & 92304.0 & 207684.0 & 9.3 & 41.0 \\
\hline 1 & 9934.2 & 5068.5 & 82161.1 & 207684.0 & 8.3 & 41.0 \\
\hline 2 & 6287.0 & 6287.0 & 76059.9 & 207684.0 & 12.1 & 33.0 \\
\hline 3 & 5883.0 & 5883.0 & 67257.2 & 207684.0 & 11.4 & 35.3 \\
\hline 4 & 5329.8 & 5329.8 & 62092.1 & 207684.0 & 11.7 & 39.0 \\
\hline 5 & 5115.8 & 5115.8 & 59307.5 & 207684.0 & 11.6 & 40.6 \\
\hline
\end{tabular}

Table 9: The FOM Comparison of Fission

\begin{tabular}{|c|c|c|c|c|c|c|}
\hline \multirow{2}{*}{ Depletion Cycle } & \multicolumn{4}{|c|}{ FOM } & \multicolumn{2}{c|}{ SUBSPACE Speedup } \\
\cline { 2 - 5 } & \multicolumn{2}{|c|}{ Analog } & \multicolumn{2}{c|}{ SUBSPACE } & \multirow{2}{*}{ Fast } & Thermal \\
\cline { 2 - 5 } & Fast & Thermal & Fast & Thermal & & \\
\hline No depletion & 3066.1 & 3880.5 & 33229.4 & 207684.0 & 10.8 & 53.5 \\
\hline 1 & 3066.1 & 5068.5 & 29578.0 & 207684.0 & 9.6 & 41.0 \\
\hline 2 & 2794.2 & 4619.0 & 27381.6 & 207684.0 & 9.8 & 45.0 \\
\hline 3 & 2614.7 & 4322.2 & 16814.3 & 207684.0 & 6.4 & 48.1 \\
\hline 4 & 1918.7 & 5329.8 & 15523.0 & 207684.0 & 8.1 & 39.0 \\
\hline 5 & 1841.7 & 5115.8 & 14826.9 & 207684.0 & 8.1 & 40.6 \\
\hline
\end{tabular}

